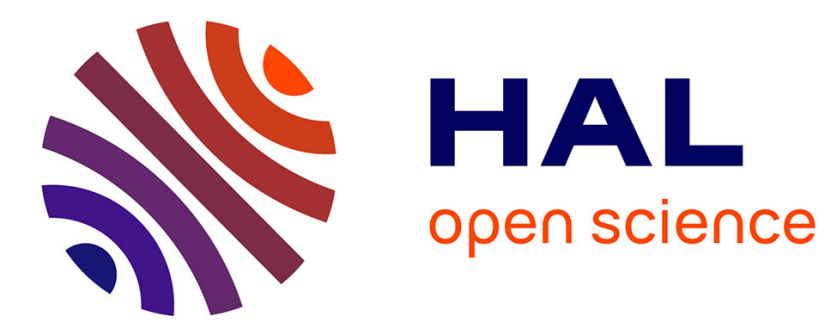

\title{
On the role of physical modelling in atmospheric and oceanic forecast \\ Joël Sommeria
}

\section{To cite this version:}

Joël Sommeria. On the role of physical modelling in atmospheric and oceanic forecast. Environmental Fluid Mechanics, 2008, Environmental Hydraulics, pp.485-493. hal-00406528

\section{HAL Id: hal-00406528 \\ https://hal.science/hal-00406528}

Submitted on 22 Jul 2009

HAL is a multi-disciplinary open access archive for the deposit and dissemination of scientific research documents, whether they are published or not. The documents may come from teaching and research institutions in France or abroad, or from public or private research centers.
L'archive ouverte pluridisciplinaire HAL, est destinée au dépôt et à la diffusion de documents scientifiques de niveau recherche, publiés ou non, émanant des établissements d'enseignement et de recherche français ou étrangers, des laboratoires publics ou privés. 


\title{
ON THE ROLE OF PHYSICAL MODELLING IN ATMOSPHERIC AND OCEANIC FORECAST
}

\author{
JOEL SOMMERIA $^{\dagger}$ \\ Coriolis-LEGI, CNRS, 21 av des Martyrs, Grenoble, France
}

\begin{abstract}
We discuss how physical modelling can be used to reproduce atmospheric or oceanic flows in the laboratory. The similarity conditions for the effects of density stratification and Earth rotation are first presented. Then examples of results obtained on the large 'Coriolis' platform in Grenoble are described. These include topographic wakes in a stratified fluid and gravity currents. Physical modelling is not used to get direct results of practical relevance, but rather to test numerical models on specific processes of environmental flows. Therefore it must be performed in close relationship with theory and numerical modelling, using advanced measurement and data assimilation techniques.
\end{abstract}

\section{Introduction}

Physical modelling is commonly used to predict environmental flows at small scale, generally for civil engineering projects. In spite of the progress in numerical modelling, it is still very relevant to get reliable flow predictions in the presence of complex geometry and topography, turbulence or multi-scale sediment dynamics.

By contrast atmospheric or oceanic forecast requires some description of the whole Earth system, which is clearly out of reach of physical modelling. It heavily relies on intensive computation and global observation systems. The global dynamics however depends on transport processes by waves and eddies at scales below the grid mesh of the forecasting models. A good characterization of these processes is essential for the reliability of global forecast. It is also needed to infer the local consequences of global predictions, for instance regional topographic effects in weather forecast.

Physical modelling can be very useful as a validation of forecasting models at these local and regional scales, typically in the range 1-1000 km, providing a detailed physical insight into the relevant fundamental processes. It complements campaigns of field measurements. For instance validation of a model by observations in the present climatic conditions is not a warranty of

\footnotetext{
†Work supported by the grant 'Hydralab' of the European Commission and by the grant ID 0527940 of the US National Science Foundation.
} 
success in the presence of radically new conditions. Furthermore, field measurements are expensive and limited in duration and spatial coverage. Natural systems are observed to change over a wide range of time scales. Distinguishing the respective influence of external perturbations and intrinsic variability is often a source of controversy. Validation by a physical model, under well controlled conditions, can provide useful clues. The engineering approach, using systematic parametric studies, brings valuable insight for natural systems.

At regional scales $(1-1000 \mathrm{~km})$ Earth curvature effects are small, so that a laboratory model with a constant gravity acceleration $\mathbf{g}$ is relevant. The density stratification and Coriolis effects must be correctly reproduced, and the corresponding non-dimensional parameters are presented in section 2 . We then show examples of physical modelling performed on the large 'Coriolis' platform in Grenoble, France, designed to reproduce both stratification and Coriolis effects in regimes at high Reynolds numbers. Therefore a variety of relevant waves and multi-scale turbulent effects can be observed, in contrast with smaller facilities. The first set of examples, presented in section 3, include wakes of topography in a stratified medium. Examples of gravity currents are discussed in section 4 .

\section{Similarity parameters}

Dynamical similarity relies on the definition of the appropriate nondimensional parameters. The most well-known is the Reynolds number,

$$
\operatorname{Re}=U H / v
$$

which is based upon the kinematic viscosity $v$, a typical velocity $U$, and a vertical scale $H$. In models of atmospheric or oceanic processes, the vertical scale $H$ is generally smaller than the horizontal scale $L$. Using the vertical scale is more relevant since friction effects are dominated by the vertical shear. The Reynolds number in the laboratory is generally much smaller than in nature, but we can expect that results would not significantly depend on Re provided it is large enough. This same hypothesis is used for usual wind tunnel tests with reduced scale models.

Density variations in the ocean are small but play a key role in the dynamics. Cold, denser, water tends to sink at the bottom and remain there, preventing vertical motion. Density stratification is also due to salinity effects in some regions. The local influence of density stratification is indicated by the buoyancy frequency $N$, which is the frequency of oscillation of a vertically displaced fluid element. 
For an incompressible fluid, $N^{2}$ is proportional to the vertical derivative $\partial \rho /(\rho \partial z)$ of the background density, $N=(-g \partial \rho /(\rho \partial z))^{1 / 2}$. For a compressible fluid, like the atmosphere, this expression has to be modified by subtracting to the vertical gradient the adiabatic gradient (the change of density associated with adiabatic vertical displacement). Otherwise the dynamics is the same as for an incompressible fluid, provided phenomena occur on limited vertical scales, over which the relative density variation is small. Therefore both oceanic and atmospheric processes can be reproduced by laboratory experiments with a similar vertical dependency of $N$. The stratification is generally produced by salinity, set by the appropriate mixture of brine and water during the initial filling of the fluid container. Evolution by diffusion is very slow, so that this initial preparation can persist for several days. The cases most commonly considered are the uniform stratification ( $N=$ cte), or the multi-layer system, made of uniform density layers separated by discontinuities. Dynamical similarity is obtained by reproducing the internal Froude number, written in terms of an average buoyancy frequency $\langle N\rangle$ as,

$$
\operatorname{Fr}=U /(<N>H)=U H^{1 / 2} /(g \delta \rho / \rho)^{1 / 2}
$$

The rotation of the reference frame, with angular velocity $\Omega$, results in centrifugal and Coriolis forces. The centrifugal force is the gradient of a potential $\left(\Phi=-\Omega^{2} r^{2} / 2\right)$ which can be added to the gravity potential. This is therefore exactly equivalent to a tilt of gravity. So the only dynamical effect of rotation is the Coriolis force, characterized by the Rossby number

$$
\mathrm{Ro}=U /(f L)
$$

where $f$ is the Coriolis parameter. It compares the vertical flow vorticity, of the order of $U / L$, to the planetary vorticity $f$. In most regions of Earth, outside the equator, the vertical component of the Coriolis force can be neglected, and the dynamics depends only on its horizontal component, proportional to $f=2 \Omega \sin \lambda$. at latitude $\lambda$. The laboratory models are generally performed in the case of weak centrifugal effects, so that $f=2 \Omega$ in the experiments. This provides a model for a region of limited extent, for which the latitude $\lambda$ can be considered constant.

Reproducing planetary scale phenomena, like planetary Rossby waves, is more problematic. A similarity exists between the effect of planetary curvature and the gradient of a layer thickness in a container with rigid lid filled with a fluid with uniform density. However this analogy does not extend to stratified cases. 

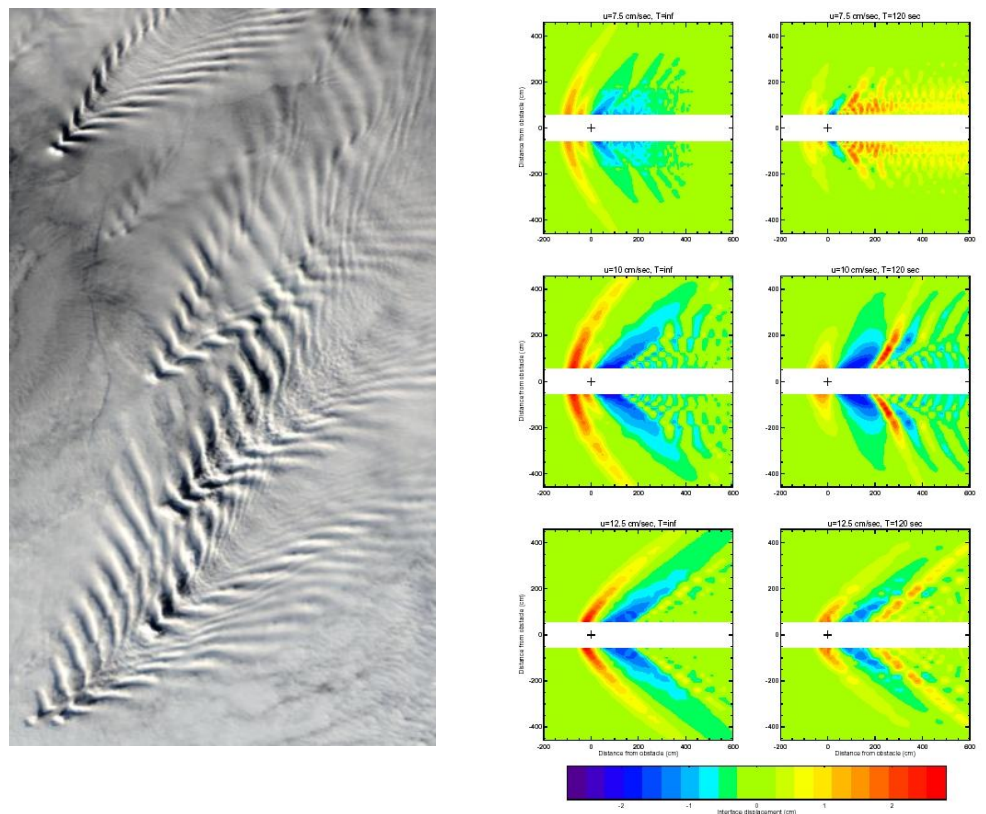

Figure 1. Internal gravity waves produced behind islands in the atmosphere (left) and in a laboratory model (right), from [5]. The color maps indicate fields of vertical displacement for the interface without Coriolis force (first column of three views), and with Coriolis force (second column). The three rows correspond respectively to a flow velocity lower, equal and higher than the wave propagation velocity. The main effect of rotation is to reduce the lateral extension, in particular for the bow wave, but it does not introduce asymmetry between the two sides in this regime (the total width of the domain is $8 \mathrm{~m}$ ).

Finally the model must be made in geometric similarity with nature, but true geometric similarity is difficult to achieve for large regions. For instance in a model of the English Channel, at scale 1/50000, the horizontal scale $L=500$ $\mathrm{km}$ is scaled down to $L=10 \mathrm{~m}$. For a true geometrical similarity, the depth $H=100 \mathrm{~m}$ should be then scaled down to $H=2 \mathrm{~mm}$. This would imply very strong, unrealistic, viscous effects. This problem is palliated by using a different scaling in the vertical and horizontal directions. Remarkably, the equation of motion is invariant by such rescaling, within the hydrostatic approximation, generally used to model large scale motion. Therefore many phenomena are preserved. However, some effects, for instance turbulent friction, are not similar. Investigating the influence of aspect ratio is therefore of high relevance, both from a practical modelling and fundamental points of view.

In summary, the physical modelling of atmospheric or atmospheric flow must reproduce the two main parameters Fr and Ro. Then an effort should be made to get large Reynolds numbers and large aspect ratio $L / H$, although it is 
generally not possible to reach the high values of natural systems. We can notice that $\operatorname{Re} \sim \operatorname{Fr} H^{3 / 2}(\delta \rho / \rho)^{1 / 2}$. Therefore the highest Re are obtained with large heights $H$ and large relative density variations $\delta \rho / \rho$. Salinity provides much stronger density variations (up to $\delta \rho / \rho=10 \%$ instead of $0.3 \%$ for temperature), so that it is more advantageous (furthermore diffusion effects are very small). The 'Coriolis' rotating platform in Grenoble provides a fairly large height $H$ (up to $1 \mathrm{~m}$ ), while providing an aspect ratio of order 10 , with a diameter $D=13 \mathrm{~m}$.

\section{3-Wakes of topography:}

Wakes over topography influence local wind conditions and airplane hazards. At a more global scale eddies and waves from topography induce friction and momentum transport and vertical mixing in the whole atmosphere. Transport effects are also induced by bottom topography in the ocean. Upwelling by submarine canyons, a process with high influence on marine life, has been modelled in laboratory[1-2].

Many laboratory experiments have been performed in the absence of rotation (e.g. Baines [3]). Local phenomena, involving boundary layers, formation of vortices with horizontal axis (rotors), vertical emission and breaking of internal gravity waves, can be observed [4].

Long-range effects involve two mechanisms, the wake of internal gravity waves on one hand, and the shedding of vortices on the other hand. The wake of internal gravity waves is commonly observed in satellite images, see figure 1 . They are visualized by the clouds produced in the zones of rising motion (due to the corresponding cooling by air expansion). Such waves can be reproduced in laboratory by horizontally translating a mountain model across a density stratified tank. An example is shown in figure 1, right side. The active fluid layer, with uniform density, is floating on a denser (salty) underlying water, providing a simple case of stratification. Maps of the interface displacement are made through a series of acoustic probes. The Coriolis effect modifies the wave structure as shown, reducing the lateral extent of the bow wave, in a symmetric way on both sides. Many other phenomena involving internal gravity waves can be studied, see e.g. [6] .

An example of vortex shedding in the atmosphere is shown in figure 2, left. It is somewhat similar to the usual Karman street of alternating vortices. However the Coriolis effect results in the stabilisation of the cyclonic vortices and a disruption of the anticyclonic ones. This asymmetry between cyclones and anticyclones is also visible in the laboratory counterpart (right), also obtained in the Coriolis platform in a fresh water layer floating on a salty underlying water. A wide variety of vortex dynamics properties can be investigated in the laboratory, with interaction of small scale three-dimensional features with larger two-dimensional eddies, in geostrophic equilibrium. 

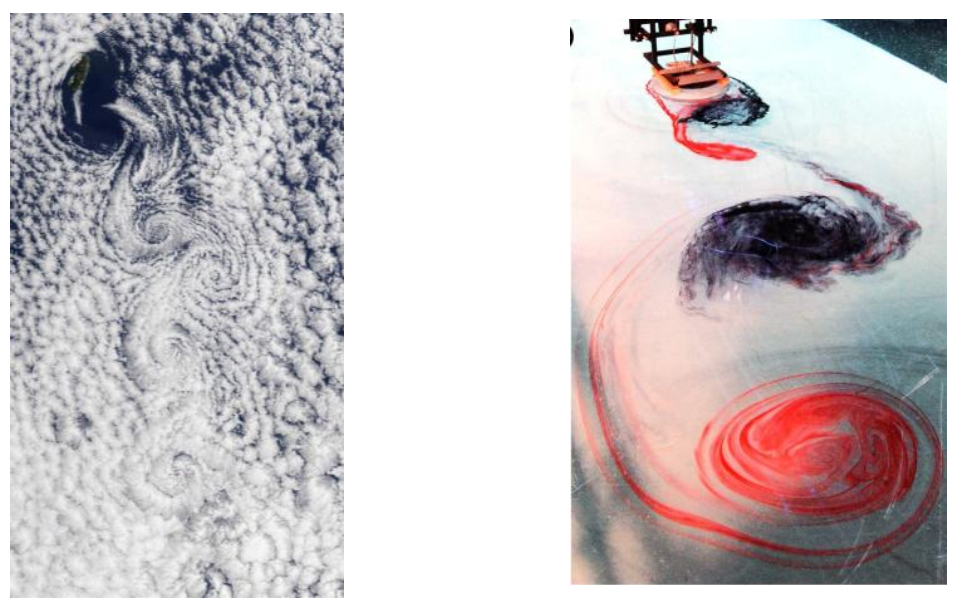

Figure 2. Eddies shed in the wake of an island (left side) and in a laboratory experiment [7] (behind a cylinder $2 \mathrm{~m}$ in diameter). The stronger coherence of cyclonic vortices (colored in red in the experiments) is observed in the appropriate range of Rossby number, like in the atmosphere.

For larger Coriolis effects, or larger scales, i.e. at smaller Rossby numbers, the distinction between internal waves and vortices is not so clear-cut. The horizontal flow associated with the gravity wave is indeed deviated by the Coriolis force, inducing vertical vorticity: this is an inertio-gravity wave. As an example, the flow over a simplified model of the Antarctic Peninsula is shown in figure 3 (right) together with a numerical computation (left), taken from Orr et al. [8]. The motivation of this study is to understand the local variations of the 40 year warming observed at different research stations around the Peninsula. The general idea is that global warming induces a weaker stratification, so that the dominant eastward wind passes over the Peninsula instead of around it. The global warming is therefore different on both sides of the Peninsula, in a way depending on the wake structure.

The laboratory experiment is designed to study this wake effect with a simple radial fixed barrier, the westward flow being produced by an initial change of angular velocity of the tank, inducing a global azimuthal flow which persists by inertia. The fluid is linearly stratified by salinity. The flow field is measured by Particle Imaging Velocity (PIV), using the correlations of two successive images of particles seeding the flow. Illumination is made by a horizontal laser sheet which scans the flow volume, as described in [9]. This provides the horizontal velocity field $\mathbf{u}$ in the volume. The vertical component $w$ is obtained from the divergence of this measured velocity, using the continuity equation, $\partial w / \partial z=-\left(\partial u_{x} / \partial x+\partial u_{y} / \partial y\right)$. 

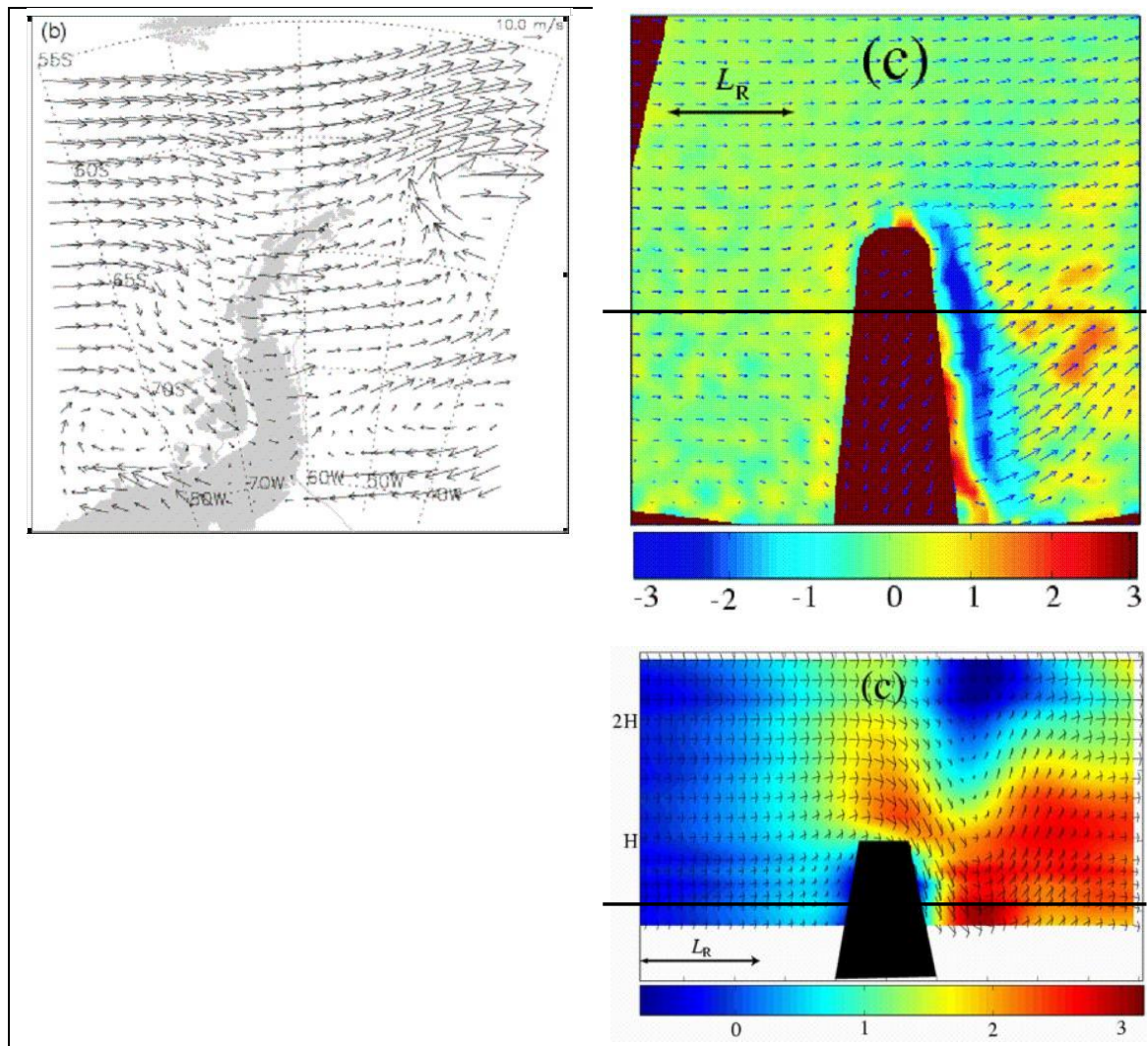

Figure 3: Flow numerically calculated around the Antarctic Peninsula (left) and physical model (right) with a simplified barrier. Horizontal (top) and vertical (bottom) cuts showing the vector field projected on the plane of cut (arrows) and the normal component as color map. The position of the vertical cut is indicated by the transverse line in the horizontal cut (from ref [8]).

From these data, horizontal and vertical cuts can be made (respectively top view and bottom views in figure 3). The velocity projection in the cut is indicated by vectors while the normal component is indicated by the color map. The horizontal cut indicates that part of the flow moves around the barrier while part is passing over it, the downward motion behind the barrier is indicated by the blue color. The wave-like motion over the barrier is also clearly visible in the vertical cut (lower view). From these data the flow rate going over and around the topography has been determined for various values of stratification (Froude number) and rotation (Rossby number), allowing us to test the numerical model. 


\section{3-Gravity currents:}

A gravity current is a flow of a fluid driven by a density difference with the surrounding background. It is produced for instance in the atmosphere by the radiative cooling of air along hill slopes. This effect of katabatic wind is particularly strong over polar caps. Many examples exist in the ocean. For instance over the Gibraltar strait, salty water from the Mediterranean Sea flows down by gravity into the Atlantic Ocean. This flow is highly turbulent, and the energy from gravity is dissipated by viscosity and partly converted into vertical mixing. Furthermore, the background is stably stratified in density, so that the flow ends up as a quasi-laminar current of mixed water in hydrostatic and geostrophic equilibrium. The rate of mixing is the key quantity to estimate in order to infer the corresponding equilibrium depth and water properties.

Reproducing gravity currents in the laboratory is fairly easy, for instance as a flow of salty water down a slope. Many experiments have been performed in sloping channels since the pioneering work of Ellison and Turner[10]. This has led to the classical model of turbulent entrainment, widely used in the modelling of natural phenomena. The effect of the Coriolis force is to laterally deviate the current, from downward to cross-slope direction, ending up in a state of geostrophic equilibrium.

An example is visualized in figure 4 . The motivation is to reproduce the overflow of dense water from the Denmark Strait which is a key process of the global thermo-haline convection, feeding the deep Atlantic Ocean with cooled surface water. Data from a measurement campaign are shown in figure 4 a, as a map of the dense water thickness. The plume is clearly deviated far from the downward direction by Coriolis effect. This has been reproduced in laboratory experiments, see $\mathrm{b}$.

Note that there can be a wide variety of gravity currents, for instance axisymmetric currents, resulting from the adjustment of a water mass on a flat bottom [14]. Other examples are surface gravity currents, deviated toward the coast by the Coriolis force [15]. The effect of canyons and other topographic features is also a subject of high interest in oceanography [16]. Convection can also occur through vertical plumes and induce planetary jets [17].

\section{4-Conclusions:}

The examples shown give a hint of the many natural flow phenomena which can be reproduced in the laboratory. The influence of density stratification and Coriolis force lead to an amazing variety of dynamical regimes. The condition of high Reynolds number makes possible a coexistence of multi-scale flow features, approaching what is observed in natural media. Meso-scale two-dimensional vortices interact with small scale turbulent eddies 
in lateral boundary layers (like island wakes), fronts or gravity currents. This multi-scale highly non-isotropic structure is a challenge for turbulence modelling. Other effects could be explored, including sediment transport and morphodynamics, thermo-haline fingering. Physical modelling allows us to study these phenomena with a control of the relevant parameters, without bias on initial or boundary conditions. Good control of the parameters provides hint on subtle questions of predictability. Finally the visual appeal of laboratory experiments helps students and research to improve their physical intuition about natural phenomena.

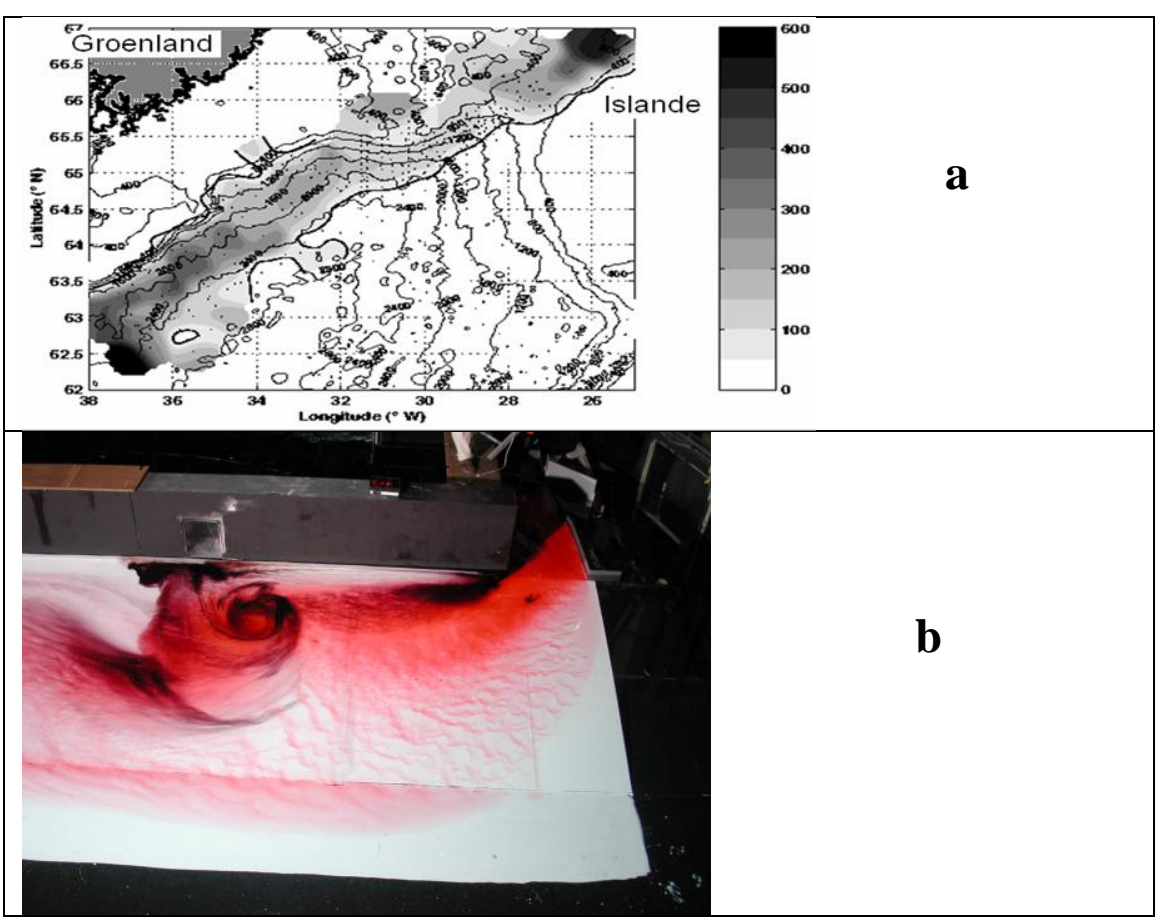

Figure 4. Denmark Strait overflow, in-situ measurement of the dense water thickness (a, from [12]) and laboratory model (b, from [13]). The dense water, colored by a red dye, is introduced at the top of the slope ( $2 \mathrm{~m}$ wide). The downward flow is deviated by the Coriolis force, while instability generates a large coherent vortex.

The goal of physical modelling in this field is not to provide direct answer to practical questions, like in most engineering practice. It is rather to improve our understanding and test our prediction capability for relevant dynamical regimes. A good understanding of the dynamics is required in the first place to establish the appropriate dynamical similarity. This may be subtle when a change of aspect ratio of horizontal and vertical scales is considered. Detailed 
comparison with numerical is provided by PIV in a volume. A still closer link between numerical model and experiments can be obtained using data assimilation [18], which allows us to initialize a model with available data in an optimum way at different stages of the flow evolution. Then the question of predictability can be addressed by measuring the further divergence of the physical and numerical models. This kind of approach could be applied to physical modelling in other fields, contributing to the needed synergy with numerical modelling and field studies [19].

\section{References}

1. D. L. Boyer and P. A. Davies, Ann. Rev. Fluid Mech. 32, 165 (2000).

2. D.L. Boyer, J. Sommeria, A.S. Mitrovic, S. Smirnov, D.B. Haidvoguel, D. Etling, J. Phys. Ocean. 36(5), 813 (2006).

3. P.G. Baines 'Topographic effects in stratified flows', Cambridge Univ. Press (1995).

4. O.S. Eiff, P Bonneton, Phys. Fluids 12, 1073 (2000).

5. E.R. Johnson, J.G. Esler, O.J. Rump, J. Sommeria, G.G. Vilenski, Proc. $R$. Soc. A 462, 3 (2006).

6. L. Gostiaux, T. Dauxois, H. Didelle, J. Sommeria, S. Viboud, Phys. Fluids 18, 056602 (2006).

7. S. Teinturier, A. Stegner, M. Ghil, S. Viboud, H. Didelle, INIST http://hdl.handle.net/2042/15598.

8. A. Orr, G.J. Marshall, J.C.R. Hunt, J. Sommeria, C.G. Wang, N.P.M. van Lipzig, D. Cresswell, J.C. King, J. Atmos. Sci. 65, 1396 (2008)

9. O. Praud , J. Sommeria and A. Fincham, J. Fluid Mech 547, 389 (2006)

10. T.H. Ellison and J.S. Turner, J. Fluid Mech. 6, 423 (1959).

11. G.F. Lane-Serffand P.G. Baines, J. Phys. Ocean 30 (2), 327 (2000).

12. R. H. Käse, J. B. Girton, T. B. Sanford, J. Geophys. Res. 108, C6, 3181 (2003)

13. S. Decamp and J. Sommeria, submitted to J. Fluid Mech.

14. M.A. Hallworth, H.E. Huppert, M. Ungarish, J Fluid Mech. 447, 1 (2001)

15. P.J. Thomas P.J. and P.F Linden P.F, J. Fluid Mech. 578, 35 (2007).

16. A. Wahlin, E. Darelius, C. Cenedese, G. Lane-Serff., Deep Sea Res. I 55

(6), 737 (2008).

17. P.L. Read, Y.H. Yasuhiro, H. Yamazaki, S.R. lewis, P.D. Williams, R. Wordsworth, K. Miki-Yamazaki, J. Sommeria,n H. Didelle, J. Atmos. Sci., 64, (11), 4031 (2007).

18. M. Galmiche, J. Sommeria, P. Brasseur, J. Verron, J. Mar. Syst., 65 (1), 532 (2007).

19. A.D. van Os, R. Soulsby, J. Kirkegaard, J. Hydr. Res. 42 (4), 341 (2004) 